\title{
Synchronous Basal Cell Carcinoma and Squamous Cell Carcinoma of Nasal Vestibule With Novel Unique Variants Identified by Whole-exome Sequencing
}

\author{
IDA GHLICHLOO $^{1}$, ZHONGBO JIN $^{2}$, RUOHAO FAN ${ }^{3}$, CAILI TONG ${ }^{4,5}$, \\ PETR STAROSTIK ${ }^{2}$, JEREMY R. CHIEN ${ }^{4,5}$ and JINPING LAI ${ }^{2,6}$ \\ ${ }^{1}$ California Northstate University College of Medicine, Elk Grove, CA, U.S.A.; \\ ${ }^{2}$ Department of Pathology, Immunology and Laboratory Medicine, \\ University of Florida College of Medicine, Gainesville, FL, U.S.A.; \\ ${ }^{3}$ Department of Otolaryngology-Head and Neck Surgery, Xiangya Hospital, \\ Central South University, Changsha, P.R. China; \\ ${ }^{4}$ Department of Biochemistry and Molecular Medicine, \\ University of California Davis Health and School of Medicine, Sacramento, CA, U.S.A.; \\ ${ }^{5}$ Department of Obstetrics and Gynecology, University of California \\ Davis Health and School of Medicine, Sacramento, CA, U.S.A.; \\ ${ }^{6}$ Department of Pathology and Laboratory Medicine, \\ Kaiser Permanente Sacramento Medical Center, Sacramento, CA, U.S.A.
}

\begin{abstract}
Background/Aim: It is estimated that nonmelanoma skin cancer (NMSC), including basal cell carcinoma (BCC) and squamous cell carcinoma (SCC), affects more than 3 million Americans each year. Translation of next-generation sequencing (NGS) data into identification of new potential targets for therapeutic applications may be helpful. Wholeexome sequencing (WES) is a widely used NGS method that involves sequencing the protein-coding regions of the genome. Case Report: We report a case of a 65-year-old female smoker who was found to have two $6 \mathrm{~mm}$ lesions in her left nasal vestibule. Biopsies demonstrated synchronous BCC and SCC. The patient underwent surgical excision of both cancers with
\end{abstract}

This article is freely accessible online.

Correspondence to: Jeremy R. Chien, Ph.D., Department of Biochemistry and Molecular Medicine, University of California Davis Health and School of Medicine, Sacramento, CA 95817, U.S.A. Tel: +1 9167344766, e-mail: jrchien@ucdavis.edu and Jinping Lai, MD, Ph.D., Department of Pathology and Laboratory Medicine, Kaiser Permanente Sacramento Medical Center, Sacramento, CA 95825, U.S.A. Tel: +1 9169737260, Fax: +1 9169737283, e-mail: Jinping.x.lai@kp.org

Key Words: Synchronous primary malignancy, basal cell carcinoma, squamous cell carcinoma, nonmelanoma skin cancer, whole exome sequencing, next generation sequencing, immunohistochemistry, immunotherapy, targeted therapy, FAM5C. safe margins followed by plastic reconstruction. WES was performed on both cancers and 16 alterations including BRCA2 (p.P389S), FAM5C (S420L), KMT2A (P855L), and SMO $(L 412 F)$, as unique for BCC, and 4 alterations including TP53 (p.H179Q) and CDKN2A (p.P114L), as unique for SCC, were identified. Conclusion: We report the first documented case with unique genetic alterations in two distinct and synchronous skin BCC and SCC arising from the same nasal vestibule of a patient. This adds to the growing field of data regarding genetic variants in characterizing malignancies and potentially for targeted therapies.

Skin cancer is the most common cancer in the United States. Basal cell carcinoma (BCC) is the most common nonmelanoma skin cancer, and lesions are most commonly found on the sun-exposed areas of the head and neck (1). Risk factors for the development of $\mathrm{BCC}$ are similar to those for squamous cell carcinoma (SCC), although BCCs are more often associated with intense, intermittent exposure to ultraviolet (UV) radiation. Ultraviolet $\mathrm{B}$ (UVB) radiation increases the risk of SCC via direct carcinogenic effect of UV light on keratinocytes within the basal layer of the epidermis; UVB-induced loss of the p53 tumor suppressor gene occurs in more than $90 \%$ of SCCs (2). Exposure to arsenic, organic hydrocarbons, ionizing radiation, and cigarette smoke are associated with an increased risk for SCC. Genetic disorders, including xeroderma pigmentosum and albinism, are associated with increased risk for SCC (3). 

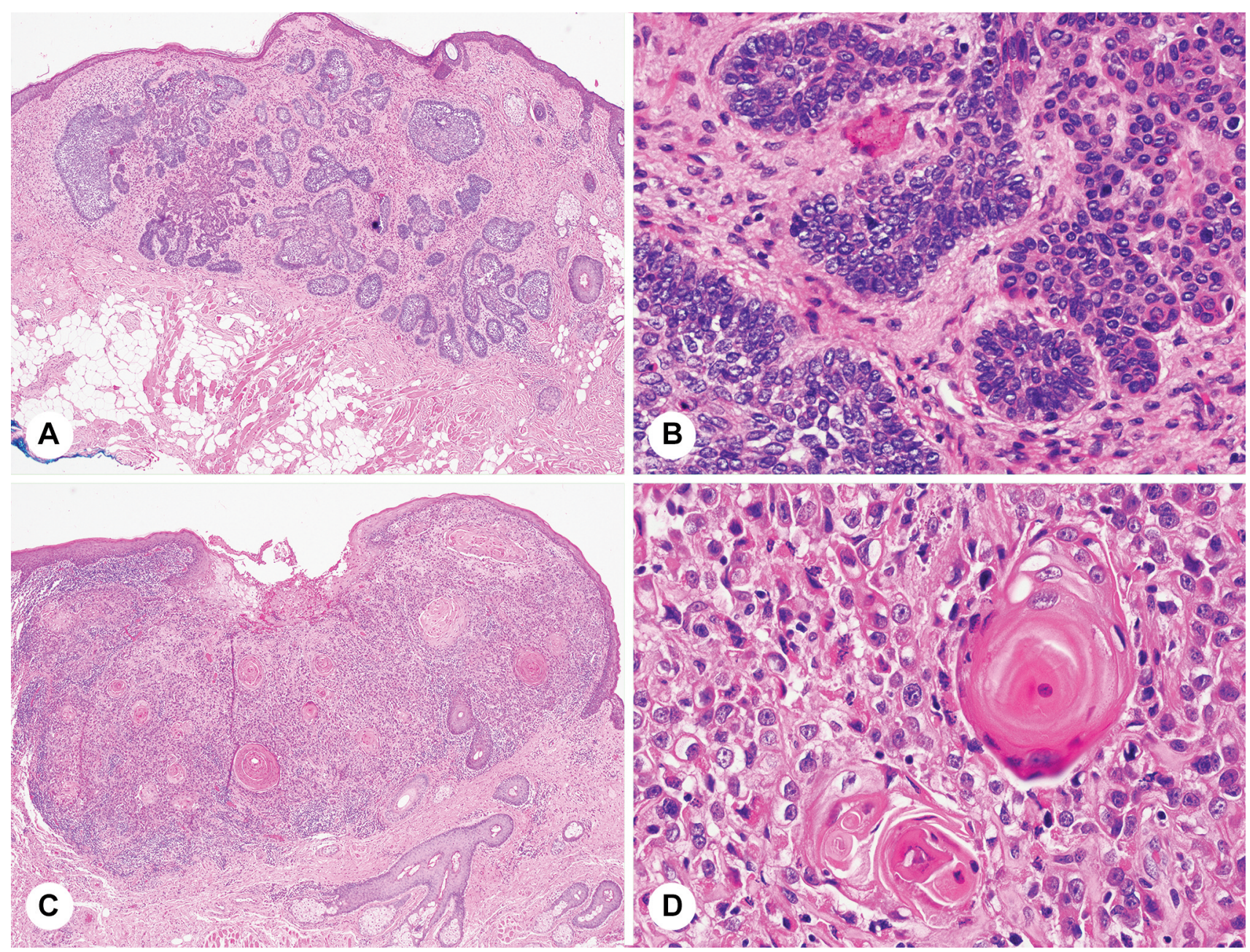

Figure 1. Synchronous nasal ala basal cell carcinoma, nodular type (A and B) and squamous cell carcinoma, well differentiated $(C$ and $D)(H \& E$ stain, $A$ and $C, 100 \times ; B$ and $D, 400 \times)$.

Immunosuppression is a well-established risk factor for SCCs of the skin, particularly with the suppression of cellmediated immunity after solid organ transplantation. Skin cancer is the most frequent malignant neoplasm in organ transplant recipients, with SCC and BCC representing $95 \%$ of these cancers. Molecular genetics has shown that the majority of BCC have somatic mutations in the PTCHI and TP53 genes (4).

Synchronous cancers are defined as two or more primary neoplasms diagnosed in a single patient within six months (5). Although synchronous $\mathrm{BCC}$ and SCC are commonly observed, cases with synchronous BCC and SCC in the same nasal vestibule have not been reported in the English literature through PubMed database search. Herein, we report a case of a 65-year-old female who was found to have synchronous primary BCC and SCC inside her left nasal vestibule. To the best of our knowledge this is the first documented case of such
BCC and SCC synchronously occurring in this anatomical location. To explore the molecular pathogenesis of the synchronous BCC and SCC, whole-exome sequencing (WES) was performed in both cancers. Interestingly, sixteen unique genetic variants including novel variant of family with sequence similarity 5 , member $\mathrm{C}$ (FAM5C) were identified uniquely to the $\mathrm{BCC}$ and four genetic variants including cyclin dependent kinase inhibitor $2 \mathrm{~A}(C D K N 2 \mathrm{~A})$ and tumor protein 53 (TP53) uniquely in SCC. The molecular pathogenesis, diagnosis, and treatment including targeted therapy of the entities are discussed.

\section{Case Report}

A 65-year-old female visited the Ear, nose and throat (ENT) clinic for lesions inside of her left nostril and on her left lateral nasal skin. She denied history of sunburn, had no past 

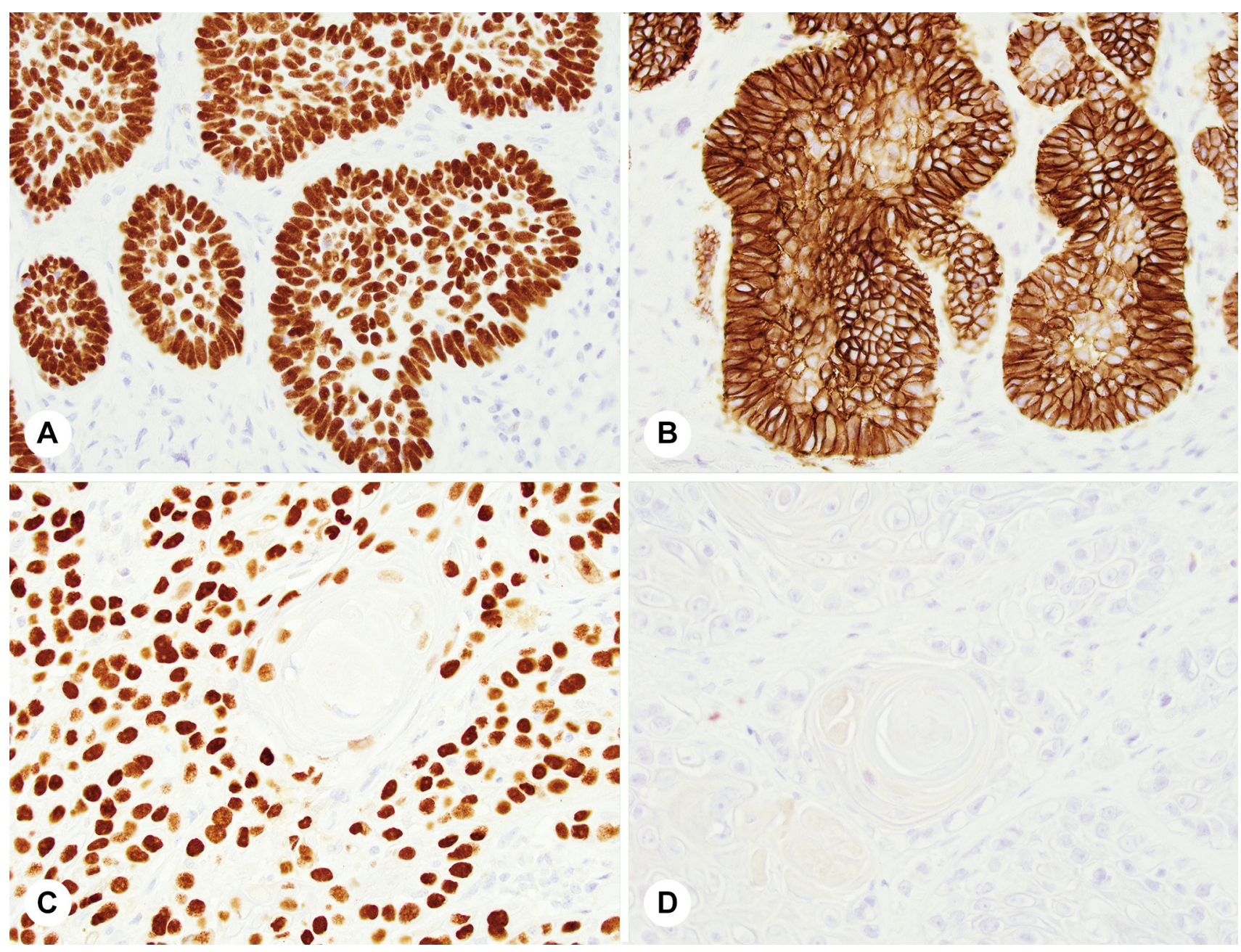

Figure 2. Immunohistochemistry shows that the basal cell carcinoma is positive for p63 (A) and Ber-EP4 (B), while squamous cell carcinoma is positive for $p 63$ (C) but negative for Ber-EP4 (D) (A-D, 400x).

surgical history and negative family history for cancers. Her social history was pertinent for a 40 -year smoking history. Rhinoscopy showed a $6 \mathrm{~mm}$ lesion on her left lateral intranasal skin and another $6 \mathrm{~mm}$ nodule on her nasal septal mucosa skin junction of the same vestibule within $2 \mathrm{~mm}$ from each other. Biopsies of both lesions revealed the left lateral intranasal skin lesion to be nodular type of $\mathrm{BCC}$ and the nodular vestibule lesion near the nasal septum to be invasive well-differentiated SCC. Excisions of left alar basal cell carcinoma and left nasal vestibule squamous cell carcinoma with resection of septal cartilage were performed. The left alar basal cell carcinoma was excised via a full thickness incision with $3 \mathrm{~mm}$ margins around the visible tumor. The left nasal vestibular squamous cell carcinoma was excised with $5 \mathrm{~mm}$ margins. This included the entire left medial nasal vestibule, anterior septum and nasal floor. Margins of the mucosa and skin were obtained and were found negative for carcinoma on the intraoperative frozen sections. Then plastic surgery was performed for cosmetic nasal reconstruction. The specimen was entirely submitted for further pathology evaluation.

In pathology, microscopically, the left nasal ala resection showed morphology of basaloid cells forming nodules with peripheral palisading consistent with nodular type of BCC (Figure 1A and $\mathrm{B}$ ). The other vestibule lesion resection revealed keratinizing squamous cell carcinoma, well differentiated (Figure 1C and D). Immunohistochemical studies demonstrated p63 immunohistochemistry-positive nuclear stains in both BCC and SCC (Figure 2A and C). BerEP4 was immunoreactive in BCC, but negative in SCC (Figure 2B and D). The patient had an uneventful postoperative recovery and was pleased with the aesthetic results of her nasal plastic reconstruction in the one-month follow-up. 
Table I. Molecular alterations unique to the synchronous basal cell carcinoma (BCC) and squamous cell carcinoma (SCC) from the same nasal vestibule.

\begin{tabular}{|c|c|c|c|}
\hline Gene & Variant at DNA level & AA Change & Cosmic ID \\
\hline \multicolumn{4}{|c|}{ Unique to $\mathrm{BCC}$} \\
\hline BCOR & NM_001123385.1:c.5073C>T & Synonymous & \\
\hline BRCA1 & NM_007300.3:c.*39G>A & & \\
\hline BRCA2 & NM_000059.3:c.1165C>T & Pro389Ser & COSV66460508 \\
\hline COL4A5 & NM_033380.2:c.2617C>T & Pro873Ser & COSV60358909 \\
\hline FAM5C & NM_199051.1:c.1259C>T & Ser420Leu COSV66526403 & \\
\hline $\mathrm{H} 3 \mathrm{~F} 3 \mathrm{~A}$ & NM_002107.4:c.*50T>A & & COSV64731835 \\
\hline KDR & NM_002253.2:c.3150_3151delCCinsTT & Arg 1051Trp & \\
\hline KDR & NM_002253.2:c.1296G>A & & \\
\hline KMT2A & NM_001197104.1:c.2564C>T & Pro855Leu & COSV 104672865 \\
\hline MSH2 & NM_000251.2:c.535C>T & Pro179Ser & \\
\hline MYH11 & NM_001040114.1:c.130G>A & Gly44Ser & \\
\hline NOTCH2 & NM_024408.3:c.4290T>G & Cys1430Trp COSV56687156 & \\
\hline NOTCH2 & NM_024408.3:c.-21_-20insCGGCGGAGG & COSV 99862555 & \\
\hline ROS1 & NM_002944.2:c.745C >T & Gln249Ter & \\
\hline SMO & NM_005631.4:c.1234C>T & Leu412Phe & COSV50824425 \\
\hline WT1 & NM_024426.4:c.94G>A & Gly32Arg & COSV60073961 \\
\hline \multicolumn{4}{|c|}{ Unique to SCC } \\
\hline CDKN2A & NM_001195132.1:c.341C >T & Pro114Leu & COSV58683051 \\
\hline MN1 & NM_002430.2:c.1544C>T & Pro515Leu & \\
\hline STAG2 & NM_001042749.1:c.2565T>G & Ile855Met & \\
\hline TP53 & NM_000546.5:c.537T>G & His $179 \mathrm{Gln}$ & COSV52673406 \\
\hline
\end{tabular}

AA, Amino acid; *Functional Analysis through Hidden Markov Models (FATHMM) prediction as pathogenic.

Tumor molecular characteristics and novel variant. Wholeexome sequencing (GatorSeq, Molecular Pathology, University of Florida) was performed on both the BCC and SCC. Sixteen alterations that were found unique for BCC and four genetic alterations were unique for SCC. Among of them, breast cancer 2, early onset (BRCA2) (p.P389S), FAM5C (S420L), lysine methyltransferase 2A (KMT2A) (P855L), and smoothened, frizzled class receptor (SMO) (L412F) variants were unique for BCC. CDKN2A (p.P114L) and TP53 (p.H179Q) variants were unique for SCC. The functional analysis through Hidden Markov Models (FAHMM) predict $C D K N 2 A$ variant as pathogenic, and the COSMIC database reported 48 cases of skin cancer with this particular alteration as a somatic variant. The FAHMM predicts the variant as neutral, H179Q variant was reported to act as a dominant-negative mutant (3). Other unique molecular alterations found in the synchronous carcinomas are described in Table I.

\section{Discussion}

Occurrence of multiple skin cancers in a single patient has been reported in the past (6). However, this case is the first reported for co-existing $\mathrm{BCC}$ and SCC in ipsilateral nasal vestibule to the best of our knowledge. Point mutations at variant locations in both BCCs and SCCs were published on
COSMIC database. However, some of the variants in our case have not been published (Table I). Under expression of FAM5C has been reported in tongue squamous cell carcinomas. Our findings suggest additional variants of FAM5C gene in the nasal BCC.

Whole-exome targeted gene sequencing was performed on both BCC and SCC lesions. Sixteen alterations were unique for BCC while four genetic alterations were unique for SCC. Among the variants for SCC, CDKN2A and TP53 are known tumor suppressor genes with well-established role in cancer pathogenesis. Among the variants for BCC, FAM5C (S420L) variant was predicted by the FATHMM as pathogenic. Kuroiwa et al. has previously reported loss of heterozygosity in the microsatellite markers near FAM5C genes and reduced expression of FAM5C in tongue squamous cell carcinoma (10). They concluded that FAM5C may be a novel tumor suppressor gene in tongue SCC. Additionally, detection of FAM5C hypermethylation has been postulated to be a marker for gastric carcinoma (11). Consistent with these studies, our analysis of the Cancer Genome Atlas data through cBioPortal webserver indicates that approximately $18 \%$ of skin cutaneous melanoma samples harbor mutations in FAM5C/BRINP3 (Figure 3). FAM5C mutations are also found in approximately $12 \%$ of cases in lung adenocarcinomas and lung squamous cell carcinomas (Figure 3). Established BCC-associated genes are Hedgehog pathway 


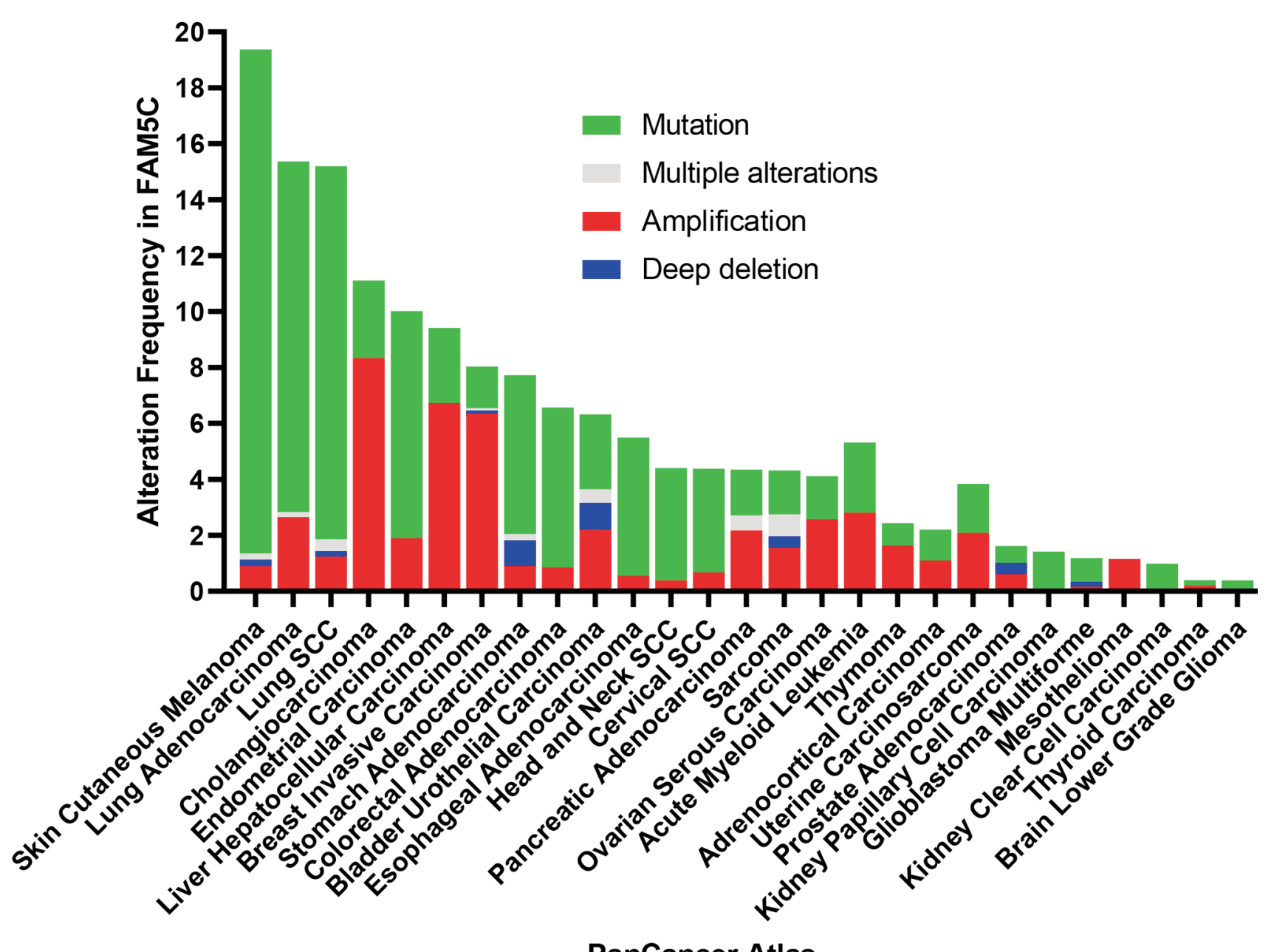

Figure 3. Somatic alterations in FAM5C reported in the Cancer Genome Atlas PanCancer studies. Specific types of alterations and frequencies of these alterations in FAM5C for each cancer type are plotted.

genes. A review of the current literature revealed a few novel BCC-associated genes: Hippo-YAP, MYCN, TERT, DPH3OXNAD1. viSMOdegib and sonidegib are immunomodulators that inhibit the Hedgehog pathway by targeting the SMO protein. Both are Food \& Drug Administration (FDA) approved for treating advanced BCC. Unfortunately, acquired resistance to SMO inhibitors has been reported in some cases (4). Due to the dynamic nature of cancer, we are hoping to bring to light more therapeutic targets via increasing the genetic knowledge of synchronous BCC and SCC. The exact role of these genetic alterations in the molecular pathogenesis of $\mathrm{BCC}$ and $\mathrm{SCC}$ remains to be elucidated for further targeted therapy.

In the BCC, the SMO (p.L412F) variant is predicted to be pathogenic by the FATHMM in the COSMIC database, and the variant is found in 11 cases of skin cancer. Other variants in BRCA2, H3 histone family member $3 \mathrm{~A}(H 3 F 3 A)$, and
Notch receptor 2 (NOTCH2) (cosmic ID COSV56687156) are predicted to be neutral. Certain mutated genes from this case have been found to be implicated in non-melanoma skin cancers in previous studies. The MSK-IMPACT study reported ROS proto-oncogene 1, receptor tyrosine kinase (ROS1), kinase insert domain receptor $(K D R), S M O, B R C A l$ mutations in BCC. The study also reported TP53 and stromal antigen 2 (STAG2) mutations in cutaneous SCC (12). A study by Abi Karam et al. focused on a cohort of BCC in 28 young patients, reported frequent mutations in TP53 (21.4\%) and SMO (10.7\%) genes (13). Although some of these somatic alterations are predicted to be pathogenic, their role in pathogenesis of BCC or SCC remains to be further elucidated.

Treatment for skin BCC and SCC is standard excision with 4-6 mm margins to a depth of the mid- or deep subcutaneous adipose tissue $(7,8)$. In our case, a complete resection of both carcinomas with safe margins and a plastic 
reconstruction were performed with aesthetic results. For patients who wish to not undergo surgery clinically safe topical agents like imiquimod, and fluorouracil 5\% cream are approved treatments as well. However, 15 percent or 35 percent of patients with history of BCC subsequently develop another primary BCC within one year or five years after diagnosis, respectively (9). Recently, inhibitors of the mammalian target of rapamycin (mTORis) exert antitumor effects and are used in organ-transplant recipients (OTR) as immunosuppressants able to reduce skin tumor burden, and the mTORis do not significantly change the immunohistochemical expression of molecules upstream of the mTOR inhibition in cutaneous SCC (14).

In summary, this report documents a rare and unusual case of synchronous primary SCC and BCC identified in the same nasal vestibule of a 65-year-old female. Biopsies with histological assessment are the key to the correct diagnosis because of the differences in tumor morphology. A complete resection of both carcinomas with safe margins and a plastic reconstruction was performed with aesthetically pleasing results. Because these two lesions were not in sun-exposed areas of the body, this shows that clinicians and pathologists need to take extra care when working up such lesions. Whole-exome sequencing performed on both cancers revealed unique point alterations in different skin cancers arising from the same nasal vestibule. The unique point alteration of FAMSC is currently of undetermined significance. This adds to the growing field of data regarding genetic variants in characterizing malignancies and potentially for targeted therapies.

\section{Conflicts of Interest}

The Authors declare that they have no conflicts of interest regarding this study.

\section{Authors' Contributions}

I.G. wrote and finalized the article; Z.J. and P.S. collected and analyzed the data; R.F. and C.T. critically reviewed the article; J.R.C critically reviewed the article, analyzed the data, made the Figure 3 and finalized the article; and J.L. designed the study, reviewed the slides, made the diagnosis, collected and analyzed the data, and finalized the article.

\section{References}

1 Nakayama M, Tabuchi K, Nakamura Y and Hara A: Basal cell carcinoma of the head and neck. J Skin Cancer 2011: 496910, 2011. PMID: 21209728. DOI: 10.1155/2011/496910

2 D'Orazio J, Jarrett S, Amaro-Ortiz A and Scott T: UV radiation and the skin. Int J Mol Sci 14(6): 12222-12248, 2013. PMID: 23749111. DOI: $10.3390 /$ ijms140612222

3 Choquet H, Ashrafzadeh S, Kim Y, Asgari MM and Jorgenson E: Genetic and environmental factors underlying keratinocyte carcinoma risk. JCI Insight 5(10): e134783, 2020. PMID: 32434987. DOI: $10.1172 /$ jci.insight.134783

4 Pellegrini C, Maturo MG, Di Nardo L, Ciciarelli V, Gutiérrez García-Rodrigo $\mathrm{C}$ and Fargnoli MC: Understanding the molecular genetics of basal cell carcinoma. Int J Mol Sci 18(11): 2485, 2017. PMID: 29165358. DOI: 10.3390/ijms 18112485

5 Cao C, Parikh P, Moezardalan K, Anantharaman A, Azarm A and Lai J: Synchronous primary pancreatic ductal carcinoma and colonic adenocarcinoma present in a patient with history of skin squamous cell carcinoma. Anticancer Res 40(7): 4029-4032, 2020. PMID: 32620648. DOI: 10.21873/anticanres.14398

6 Darlington D, Puthanmadhom Narayanan S and Anitha FS: Synchronous triple malignancies in an Indian albino: A case report. Cureus 10(8): e3190, 2018. PMID: 30364818. DOI: $10.7759 /$ cureus. 3190

7 Stratigos A, Garbe C, Lebbe C, Malvehy J, del Marmol V, Pehamberger H, Peris K, Becker JC, Zalaudek I, Saiag P, Middleton MR, Bastholt L, Testori A, Grob JJ, European Dermatology Forum (EDF), European Association of DermatoOncology (EADO) and European Organization for Research and Treatment of Cancer (EORTC): Diagnosis and treatment of invasive squamous cell carcinoma of the skin: European consensus-based interdisciplinary guideline. Eur J Cancer 51(14): 1989-2007, 2015. PMID: 26219687. DOI: 10.1016/ j.ejca.2015.06.110

8 Gulleth Y, Goldberg N, Silverman RP and Gastman BR: What is the best surgical margin for a Basal cell carcinoma: a metaanalysis of the literature. Plast Reconstr Surg 126(4): 1222-1231, 2010. PMID: 20885244. DOI: 10.1097/PRS.0b013e3181ea450d

9 Wehner MR, Linos E, Parvataneni R, Stuart SE, Boscardin WJ and Chren MM: Timing of subsequent new tumors in patients who present with basal cell carcinoma or cutaneous squamous cell carcinoma. JAMA Dermatol 151(4): 382-388, 2015. PMID: 25588079. DOI: 10.1001/jamadermatol.2014.3307

10 Kuroiwa T, Yamamoto N, Onda T and Shibahara T: Expression of the FAM5C in tongue squamous cell carcinoma. Oncol Rep 22(5): 1005-1011, 2009. PMID: 19787213. DOI: 10.3892/ or_00000528

11 Chen L, Su L, Li J, Zheng Y, Yu B, Yu Y, Yan M, Gu Q, Zhu Z and Liu B: Hypermethylated FAM5C and MYLK in serum as diagnosis and pre-warning markers for gastric cancer. Dis Markers 32(3): 195-202, 2012. PMID: 22377736. DOI: 10.3233/DMA-2011-0877

12 Zehir A, Benayed R, Shah RH, Syed A, Middha S, Kim HR, Srinivasan P, Gao J, Chakravarty D, Devlin SM, Hellmann MD, Barron DA, Schram AM, Hameed M, Dogan S, Ross DS, Hechtman JF, DeLair DF, Yao J, Mandelker DL, Cheng DT, Chandramohan R, Mohanty AS, Ptashkin RN, Jayakumaran G, Prasad M, Syed MH, Rema AB, Liu ZY, Nafa K, Borsu L, Sadowska J, Casanova J, Bacares R, Kiecka IJ, Razumova A, Son JB, Stewart L, Baldi T, Mullaney KA, Al-Ahmadie H, Vakiani E, Abeshouse AA, Penson AV, Jonsson P, Camacho N, Chang MT, Won HH, Gross BE, Kundra R, Heins ZJ, Chen HW, Phillips S, Zhang H, Wang J, Ochoa A, Wills J, Eubank M, Thomas SB, Gardos SM, Reales DN, Galle J, Durany R, Cambria R, Abida W, Cercek A, Feldman DR, Gounder MM, Hakimi AA, Harding JJ, Iyer G, Janjigian YY, Jordan EJ, Kelly CM, Lowery MA, Morris LGT, Omuro AM, Raj N, Razavi P, Shoushtari AN, Shukla N, Soumerai TE, Varghese AM, Yaeger R, Coleman J, Bochner B, Riely GJ, Saltz LB, Scher HI, 
Sabbatini PJ, Robson ME, Klimstra DS, Taylor BS, Baselga J, Schultz N, Hyman DM, Arcila ME, Solit DB, Ladanyi M and Berger MF: Mutational landscape of metastatic cancer revealed from prospective clinical sequencing of 10,000 patients. Nat Med 23(6): 703-713, 2017. PMID: 28481359. DOI: 10.1038/ nm.4333

13 Abi Karam M, Kourie HR, Jalkh N, Mehawej C, Kesrouani C, Haddad FG, Feghaly I, Chouery E and Tomb R: Molecular profiling of basal cell carcinomas in young patients. BMC Med Genomics 14(1): 187, 2021. PMID: 34284772. DOI: 10.1186/ s12920-021-01030-w
14 Koletsa T, Petrakis G, Karayannopoulou G, Euvrard S and Kanitakis $\mathrm{J}$ : mTOR signalling pathway-protein expression in post-transplant cutaneous squamous-cell carcinomas before and after conversion to mTOR-inhibitors. Anticancer Res 38(6): 3319-3322, 2018. PMID: 29848679. DOI: 10.21873/anticanres. 12597

Received August 22, 2021

Revised September 18, 2021

Accepted October 7, 2021 